\title{
Anillos totales de fracciones y anillos de Hermite
}

\author{
Total rings of fractions and Hermite rings
}

\author{
C. Granados Pinzón ${ }^{\mathrm{a}, *}$ \\ W. Olaya León ${ }^{\mathrm{b}, *}$
}

\author{
Fecha de Recepción: 03.02.2020 \\ Fecha de Aceptación: 17.05.2020 \\ Doi: https://doi.org/10.19053/01217488.v11.n2.2020.10223
}

\section{Resumen}

En este artículo se estudian propiedades generales de los anillos totales de fracciones y los anillos de Hermite. Por otra parte se encuentra una relación entre estos anillos y las $K$-álgebras finitas. Una $K$-álgebra finita es una álgebra conmutativa con unidad de dimensión finita como espacio vectorial sobre un cuerpo $K$. Más exactamente, se prueba que las $K$-álgebras finitas son anillos totales de fracciones y anillos de Hermite. Además, se muestra que el producto directo de cuerpos es también ejemplo de anillo total de fracciones y anillo de Hermite.

Palabras clave: Localización, producto directo de anillos, anillo de Hermite y $K$-álgebra finita..

\begin{abstract}
In this paper, we show general properties of total rings of fractions and of Hermite rings. We study the relationships between those rings and the finite dimensional $K$-algebras. A finite dimensional $K$-algebra is a commutative algebra with unit such that this is finite dimensional as vector space over a field $K$. We proof that the finite dimensional $K$-algebras are total rings of fractions and also Hermite rings. In addition, we show that direct product of fields is another example of total ring of fractions and Hermite ring.
\end{abstract}

Key words: Localization, direct product of rings, Hermite ring and finite dimensional $K$-algebra.

\footnotetext{
${ }^{a}$ Escuela de Matemáticas, Universidad Industrial de Santander, Bucaramanga, Colombia.

*Correo electrónico: cigranad@uis.edu.co

${ }^{\mathrm{b}}$ Escuela de Matemáticas, Universidad Industrial de Santander, Bucaramanga, Colombia.

*Correo electrónico: wolaya@uis.edu.co
} 


\section{Introducción}

La construcción de los anillos de fracciones es similar a la de los números racionales a partir de los números enteros, desde el punto de vista del álgebra conmutativa esta construcción tiene mucha importancia porque relaciona dos áreas de la matemática, el álgebra y la geometría.

En 1926, H. Grell, un alumno de E. Noether, definió el anillo de fracciones de un dominio entero; su extensión a anillos noetherianos se dio en 1944 por Chevalley y en 1948, Uzkov la definió en el caso general [4]. En adelante, se entiende por anillo a un anillo conmutativo con unidad. Sean $R$ un anillo y $S$ un subconjunto multiplicativo de $R$, es decir, $1 \in S$ y si $s, t \in S$ entonces $s t \in S$. Se define en $R \times S$ la relación de equivalencia $(f, g) \sim(u, v) \Leftrightarrow$ $(f v-g u) s=0$ para algún $s \in S$. En el conjunto cociente $R_{S}:=(R \times S) / \sim$, se denota a la clase de equivalencia de $(f, g)$ como $\frac{f}{g}$ y se definen las operaciones suma y producto así:

$$
\frac{f}{g}+\frac{u}{v}=\frac{f v+g u}{g v} \quad \text { y } \quad \frac{f}{g} \frac{u}{v}=\frac{f u}{g v} .
$$

Estas operaciones están bien definidas y hacen de $R_{S}$ un anillo conmutativo con unidad, donde el cero es $\frac{0}{s}$, para $s \in S$ y la unidad es $\frac{s}{s}$, para $s \in S$. Más aún, se tiene un homomorfismo canónico de anillos $\varphi: R \rightarrow R_{S}$ dado por $\varphi(f):=\frac{f}{1}$ que en general no es inyectivo. El anillo $R_{S}$ se llama anillo de fracciones o localización de $R$ por $S$. La construcción anterior generaliza la construcción del cuerpo de los números racionales $\mathbb{Q}$ a partir del dominio de los números enteros $\mathbb{Z}$, donde $S=\mathbb{Z}-\{0\}$. En general, si $R$ es un dominio entero y $S=R-\{0\}$, entonces $S$ es un subconjunto multiplicativo y $R_{S}:=K(R)$ es el cuerpo de fracciones de $R$. En este caso, el morfismo canónico $\varphi: R \rightarrow K(R)$ es inyectivo [1, 3, 5, 14]. Si $\mathfrak{p}$ es un ideal primo de $R$, entonces $S=R-\mathfrak{p}$ es un subconjunto multiplicativo y en este caso se usa la notación $R_{S}=R_{\mathfrak{p}}$. Si $S_{0}$ es el subconjunto de los no divisores de cero de un anillo $R$, entonces $S_{0}$ es un subconjunto multiplicativo y el anillo $S_{0}^{-1} R:=R_{S_{0}}$ es llamado anillo total de fracciones de $R$.

La relación del anillo total de fracciones con otros anillos es un tema de investigación en álgebra conmutativa [2, 10]. En este artículo se estudia la relación de los anillos totales de fracciones con los dominios euclídeos, los productos directos de cuerpos y las
$K$-álgebras finitas. Se entiende por $K$-álgebra finita a una álgebra conmutativa con unidad y de dimensión finita como $K$-espacio vectorial. Además, los anillos de Hermite son de interés permanente entre los investigadores para resolver la conjetura relacionada con estos anillos [12, 13, 15, 16, 17]. Aquí se muestra que los anillos locales, las $K$-álgebras finitas y el producto directo de cuerpos son anillos de Hermite. Nuestro interés por estos anillos se basa en un problema abierto en geometría proyectiva, el cual consiste en caracterizar la recta proyectiva sobre anillos [6, 9, 11]. Más exactamente, queremos caracterizar las rectas proyectivas sobre anillos totales de fracciones y sobre anillos de Hermite. En dirección a este fin, se han estudiado las $K$-álgebras finitas en [7] y [6] es un estudio inicial de la recta proyectiva sobre anillos totales de fracciones.

En la sección siguiente se define el anillo total de fracciones de forma un poco distinta a la enunciada arriba, se muestra la relación de estos anillos con los dominios euclídeos y se prueba que el producto directo de anillos totales de fracciones es un anillo total de fracciones. En la tercera sección se estudian los anillos de Hermite y se muestra que los anillos locales y el producto directo de anillos de Hermite son anillos de Hermite. En consecuencia, se tiene que el producto directo de cuerpos es ejemplo tanto de anillo total de fracciones como anillo de Hermite. Finalmente, en la cuarta sección se muestra que las $K$-álgebras finitas son anillos totales de fracciones y también anillos de Hermite.

\section{Anillo total de fracciones}

Definición 2.1. Un anillo $R$ es un anillo total de fracciones si sus elementos son invertibles o divisores de cero.

Ejemplo 2.2. Un cuerpo es un anillo total de fracciones y un dominio entero que no es un cuerpo no es anillo total de fracciones. En particular, el anillo $K[x]$ de polinomios con coeficientes en un cuerpo $K$ no es un anillo total de fracciones, pues $x$ no es ni invertible ni divisor de cero en $K[x]$.

Definición 2.3. Se dice que un anillo $R$ es un dominio euclídeo si $R$ es un dominio entero y existe una aplicación $\delta: R-\{0\} \rightarrow \mathbb{N}$ tal que

$$
\text { 1. } \delta(a) \leq \delta(a b) \text { para todos } a, b \in R-\{0\} .
$$




\section{Granados Pinzón - W. Olaya León}

2. Si $a \in R-\{0\}$, para cada $b \in R$ existen $c, r \in$ $R$ tales que $b=a c+r, \delta(r)<\delta(a)$ ó $r=0$.

Ejemplo 2.4. Los siguientes son ejemplos de anillos ecuclídeos:

1. El anillo de números enteros $\mathbb{Z}$ con $\delta(n)=|n|$,

2. El anillo $K[x]$ de polinomios con coeficientes en el cuerpo $K \operatorname{con} \delta(p(x))=\operatorname{gr}(p(x))$.

Como todo ideal de un anillo euclídeo $R$ es principal, entonces $f R+g R=d R$ si $f, g \in R$ y $d=\operatorname{mcd}(f, g)$. Luego, se tiene la identidad de Bézout.

\section{Identidad de Bézout:}

$$
d=\lambda f+\mu g \text { donde } \lambda, \mu \in R \text {. }
$$

La Proposición 2.5 relaciona el dominio euclídeo con el anillo total de fracciones y permite mostrar otros ejemplos.

Proposición 2.5. Si $R$ es un dominio euclídeo y $0 \neq f \in R$, entonces $R /(f)$ es un anillo total de fracciones.

Demostración. Sea $g+(f) \in R /(f)$ y supongamos que $d=\operatorname{mcd}(f, g)$. Por la identidad de Bézout, existen $\lambda, \mu \in R$ tales que $\lambda f+\mu g=d$. Por tanto,

$$
\begin{aligned}
(g+(f))(\mu+(f)) & =g \mu+(f) \\
& =g \mu+\lambda f+(f)=d+(f) .
\end{aligned}
$$

Consideremos los dos casos siguientes:

1. Si $d$ es invertible en $R$, entonces $d+(f)$ es invertible en $R /(f)$ y por tanto $g+(f)$ también lo es.

2. Si $d$ no es invertible, como $d \mid f$ y $d \mid g$, existen $c_{1}, c_{2} \in R$ tales que $f=c_{1} d, g=c_{2} d$. Luego $c_{1}+(f) \neq 0$ ya que $f$ no divide a $c_{1}$ pues $d$ no es invertible. Ahora, como $(g+(f))\left(c_{1}+(f)\right)=$ $g c_{1}+(f)=c_{2} d c_{1}+(f)=0+(f)$, entonces $g+(f)$ es divisor de cero.

Por la Proposición 2.5 se tienen los ejemplos:

1. Consideremos el anillo $\mathbb{Z}$ y $0 \neq n \in \mathbb{Z}$. Entonces el anillo $\mathbb{Z} /(n)$ es un anillo total de fracciones.
2. Sea $K[x]$ el anillo de polinomios con coeficientes en el cuerpo $K$. Entonces los anillos $K[x] /(f(x))$, con $f(x) \neq 0$, son anillos totales de fracciones, en particular, el cuerpo complejo

$$
\mathbb{C}=\frac{\mathbb{R}[x]}{\left(x^{2}+1\right)}=\left\{a+b i: a, b \in \mathbb{R}, i^{2}=-1\right\},
$$

el anillo de los paracomplejos

$$
\mathbb{P}=\frac{\mathbb{R}[x]}{\left(x^{2}-1\right)}=\left\{a+b j: a, b \in \mathbb{R}, j^{2}=1\right\}
$$

y el anillo de los números duales

$$
\mathbb{D}=\frac{\mathbb{R}[x]}{\left(x^{2}\right)}=\left\{a+b \varepsilon: a, b \in \mathbb{R}, \varepsilon^{2}=0\right\}
$$

son anillos totales de fracciones.

Proposición 2.6. Si $R$ es un anillo total de fracciones, entonces

$$
A=\frac{R[x]}{\left(x^{2}\right)}=\left\{a+b \gamma: a, b \in R, \gamma^{2}=0\right\}
$$

es anillo total de fracciones.

Demostración. Sean $a+b \gamma, c+d \gamma \in A$. Por la fórmula del producto,

$$
(a+b \gamma)(c+d \gamma)=a c+(b c+a d) \gamma .
$$

Como $R$ es anillo total de fracciones, tenemos dos posibilidades:

1. $a+b \gamma \in A$ es invertible si y sólo si $a \in R$ es invertible. En efecto, si $a \in R$ es invertible entonces existen $c, d \in R,(c, d) \neq(0,0)$ tales que $a c=1 \mathrm{y} b c+a d=0$, luego $a$ es invertible en $R$. Recíprocamente, si $a$ es invertible en $R$,

$$
(a+b \gamma)\left(a^{-1}-b a^{-2} \gamma\right)=1 .
$$

Luego $a+b \gamma$ es invertible.

2. $a+b \gamma \in A$ es divisor de cero si y sólo si $a \in R$ es divisor de cero. En efecto, si $a+b \gamma \in A$ es divisor de cero, existen $c, d \in R,(c, d) \neq(0,0)$ tales que $a c=b c+a d=0$. Así, si $c$ es divisor de cero, $a$ es divisor de cero de $R$; y si $c=0$ entonces $a d=0$ luego $a$ es divisor de cero. Recíprocamente, si $a$ es divisor de cero en $R$, existe $c \neq 0$ con $a c=0$ y $b c+a d=0$. En consecuencia, $a+b \gamma \in A$ es divisor de cero. 
2. Existe un homomorfismo

Proposición 2.7. Sean $R$ un anillo, $S \subset R$ un subconjunto multiplicativo y $S^{-1} R=\left\{\frac{a}{b}: a \in R\right.$ y $\left.b \in S\right\}$. Consideremos el homomorfismo canónico

$$
\begin{aligned}
& \varphi: \quad R \rightarrow S^{-1} R \\
& a \mapsto \quad \frac{a}{1}
\end{aligned}
$$

Entonces

1. $\frac{a}{s} \in S^{-1} R$ es invertible si y sólo si existen $b \in$ $R$ у $u \in S$ con $a b u \in S$.

2. $\frac{a}{s} \in S^{-1} R$ es divisor de cero si y sólo si existe $b \in R$ tal que

(i) $b v \neq 0$ para todo $v \in S$,

(ii) existe $u \in S$ con $a b u=0$.

3. $a \in \operatorname{Ker}(\varphi)$ si y sólo si existe $s \in S$ con $a s=0$.

Demostración. 1. $\frac{a}{s} \in S^{-1} R$ es invertible si y sólo si existe $\frac{b}{t} \in S^{-1} R$ tal que $\frac{a}{s} \frac{b}{t}=1$ y esto equivale a que existe $u \in S$ tal que $(a b-s t) u=0$ luego $a b u=s t u \in S$. Recíprocamente, si $v:=a b u \in S$ entonces $\frac{a}{s} \frac{b u s}{v}=1$.

2. $\frac{a}{s} \in S^{-1} R$ es divisor de cero si y sólo si existe $\frac{b}{t} \neq 0$ tal que $\frac{a}{s} \frac{b}{t}=0$ y esto equivale a que existe $u \in S$ tal que $a b u=0$ y $b v \neq 0$ para todo $v \in S$.

3. Inmediato.

Observación 2.8. Si $a$ es invertible en $R$, lo es en $S^{-1} R$ porque $a a^{-1}(1)=1 \in S$. Pero si $a$ es divisor de cero en $R$ no necesariamente lo es en $S^{-1} R$ e incluso puede ser invertible en este anillo. Por ejemplo, en $R=\mathbb{Z} /(6), \overline{2}$ es divisor de cero en $R$ pero si $S=$ $\{\overline{1}, \overline{2}, \overline{4}\}$ entonces $S$ es un subconjunto multiplicativo y $\overline{2} \in S$ luego $\overline{2}$ es invertible en $S^{-1} R=\{\overline{0}, \overline{1}, \overline{2}\}$. Recíprocamente, $a$ puede ser invertible en $S^{-1} R$ sin serlo en $R$ y si $a$ es divisor de cero en $S^{-1} R$ entonces $a$ es divisor de cero en $R$.

Proposición 2.9. Sea $S$ el subconjunto multiplicativo de los no divisores de cero de un anillo $R$. Consideremos el anillo $\Sigma=S^{-1} R=\left\{\frac{a}{b}: a \in R, b \in S\right\}$ entonces

1. $\Sigma$ es un anillo total de fracciones.

$$
\begin{aligned}
\varphi: & R \rightarrow \Sigma \\
a & \mapsto \frac{a}{1}
\end{aligned}
$$

tal que para todo anillo total de fracciones $\Delta$ y todo homomorfismo $f: R \rightarrow \Delta$ existe un único homomorfismo $\bar{f}: \Sigma \rightarrow \Delta$ con el cual el diagrama siguiente es conmutativo:

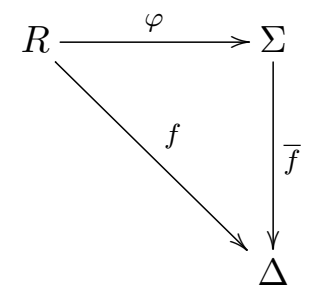

Demostración. 1. Sea $z=\frac{a}{b} \in \Sigma$. Si $a \in S$ entonces $a(1)(1) \in S$ luego, por la Proposición 2.7. $z$ es invertible. Si $a \notin S$ entonces $a$ es divisor de cero, luego existe $0 \neq c \in R$ tal que $a c=0$. Así, $a c 1=0$ y $c s \neq 0$ para todo $s \in S$ pues los elementos de $S$ no son divisores de cero luego, por la Proposición 2.7, $z$ es divisor de cero.

2. Es consecuencia de la propiedad universal de los anillos de fracciones ya que todo homomorfismo unitario de anillos transforma elementos invertibles en invertibles.

El homomorfismo $\varphi$, de la Proposición 2.9, es inyectivo. En efecto, si $\varphi(a)=\frac{a}{1}=0$, existe $b \in S$ tal que $a b=0$ pero como $S$ está formado por los elementos no divisores de cero entonces $a=0$.

Definición 2.10. El anillo $\Sigma=S^{-1} R=\left\{\frac{a}{b}: a \in\right.$ $R, b \in S\}$, dado en la Proposición 2.9, se llama anillo total de fracciones de $R$.

Proposición 2.11. Un anillo $\Sigma$ es anillo total de fracciones si y sólo si existe $R$, no necesariamente único, tal que $\Sigma$ es el anillo total de fracciones de $R$.

Demostración. Si $\Sigma$ es un anillo total de fracciones entonces $\Sigma$ es el anillo total de fracciones de $\Sigma$. Recíprocamente, si $\Sigma$ es el anillo total de fracciones de un anillo $R$, por la Proposición 2.9, $\Sigma$ es un anillo total de fracciones. El anillo $R$ no es único pues si $\Sigma$ es el anillo total de fracciones de un anillo $R \neq \Sigma$, se tiene que $\Sigma$ también es el anillo total de fracciones de $\Sigma$. 
El siguiente es otro ejemplo de anillo total de fracciones.

Ejemplo 2.12. Sean $K$ un cuerpo y

$$
R=K[x, y]_{(x, y)} /\left(x y, y^{2}\right) .
$$

1. $K[x, y]_{(x, y)}$ es un anillo local con ideal maximal $\mathfrak{m}=(x, y)$ donde identificamos $\frac{x}{1}=x$ y $\frac{y}{1}=y$. Note que $R$ es un anillo local con ideal maximal $\mathfrak{m}=(\bar{x}, \bar{y})$, donde $\bar{x}=x+\left(x y, y^{2}\right)$ y $\bar{y}=$ $y+\left(x y, y^{2}\right)$.

2. $R$ es un anillo total de fracciones ya que los elementos que no están en el maximal $\mathfrak{m}$ son invertibles por ser $R$ anillo local y los elementos de $\mathfrak{m}$ son divisores de cero pues existe $0 \neq \bar{y} \in$ $\mathfrak{m}$ tal que $(\alpha \bar{x}+\beta \bar{y}) \bar{y}=0$ para todos $\alpha, \beta \in R$.

3. Los elementos de $R$ admiten una escritura única como $\frac{a+\bar{x} A(\bar{x})+c \bar{y}}{b+\bar{x} B(\bar{x})+d \bar{y}} \operatorname{con} a, b, c, d \in K, b \neq 0 \mathrm{y}$ $A, B \in K[\bar{x}]$. Además los elementos de $R /(\bar{y})$ admiten una escritura única como $\frac{a+\bar{x} A(\bar{x})}{b+\bar{x} B(\bar{x})}$ con $a, b \in K, b \neq 0$ y $A, B \in K[\bar{x}]$. Note que $R /(\bar{y}) \simeq K[\bar{x}]_{(\bar{x})}$ y como $K[\bar{x}]_{(\bar{x})}$ es un dominio entero, entonces el ideal $(\bar{y})$ es primo y $(\bar{y}) \subset \mathfrak{m}$.

Observación 2.13. Si $R$ es un anillo total de fracciones y a es un ideal de $R$, entonces $R / \mathfrak{a}$ no es en general un anillo total de fracciones, por ejemplo, sea $R$ el anillo total de fracciones del Ejemplo 2.12. Como $(\bar{y})$ es un ideal primo no maximal de $R$ entonces $R /(\bar{y})$ es un dominio entero y no es cuerpo. En consecuencia, $R /(\bar{y})$ no es anillo total de fracciones.

\subsection{Producto directo de anillos}

Consideremos el producto directo de una familia de anillos $\left\{R_{i}\right\}_{i \in I}$. Es decir, dada $\left\{R_{i}\right\}_{i \in I}$, existe un anillo $R=\prod_{i \in I} R_{i}$ junto con los homomorfismos de anillos $\boldsymbol{i}_{i}: R_{i} \rightarrow R, i \in I$, tal que para todo anillo $S$ y para todos los homomorfismos $\varphi_{i}: R_{i} \rightarrow S$, existe un único homomorfismo $\varphi: R \rightarrow S \operatorname{con} \varphi_{i}=\varphi \circ \boldsymbol{i}_{i}$, ver [1, 8].

La suma directa de la familia anterior es el conjunto

$$
\bigoplus_{i \in I} R_{i}:=\left\{\left(f_{i}\right) \in \prod_{i \in I} R_{i}: \text { casi todos los } f_{i}=0\right\}
$$

donde por "casi todos" queremos decir "todos, excepto un número finito".
Observe que si el conjunto de índices $I$ es finito, entonces

$$
\bigoplus_{i \in I} R_{i}=\prod_{i \in I} R_{i}
$$

La Proposición 2.14 caracteriza los elementos invertibles y los divisores de cero de un producto directo de anillos, ver [7]. Sea $\pi_{i}: R \rightarrow R_{i}$ la proyección $i$-ésima, es decir para $\boldsymbol{f}=(f(i))_{i \in I} \in R$, $\pi_{i}(\boldsymbol{f})=f(i)$.

Proposición 2.14. Sean $R=\prod_{i \in I} R_{i}$ y $\boldsymbol{f}=$ $(f(i))_{i \in I} \in R$. Entonces

(1) $f$ es invertible si y sólo si, para todo $i \in I$, $\pi_{i}(\boldsymbol{f})=f(i)$ es invertible.

(2) $f$ es un divisor de cero si y sólo si existe $i \in I$ tal que $\pi_{i}(\boldsymbol{f})=f(i)$ es divisor de cero.

Demostración. Se sigue de las definiciones.

El Corolario 2.15 muestra que el producto de anillos totales de fracciones es anillo total de fracciones y, en particular, el producto directo de cuerpos es anillo total de fracciones.

Corolario 2.15. Si para todo $i \in I, R_{i}$ es un anillo total de fracciones, entonces $R=\prod_{i \in I} R_{i}$ es también anillo total de fracciones.

Demostración. Consecuencia de la Proposición 2.14 ya que $R_{i}$ es anillo total de fracciones para todo $i \in$ $I$.

Corolario 2.16. Sean $R=\prod_{i \in I} R_{i}$ y $\boldsymbol{f}=$ $(f(i))_{i \in I} \in R$. Si para todo $i \in I, R_{i}$ es un cuerpo, entonces

1. $\boldsymbol{f}$ es invertible si y sólo si $f(i) \neq 0$ para todo $i \in I$.

2. $f$ es un divisor de cero si y sólo si existe $i \in I$ tal que $f(i)=0$.

3. $R$ es un anillo total de fracciones.

Demostración. Las dos primeras afirmaciones se tienen por la Proposición 2.14 y la tercera por el Corolario 2.15

Corolario 2.17. $\mathrm{Si}$, para todo $i \in I, R_{i}$ es un anillo y $R=\prod_{i \in I} R_{i}$ es un anillo total de fracciones entonces $R_{i}$ es un anillo total de fracciones, para todo $i \in I$. 
Demostración. Consecuencia de la Proposición 2.14 ya que $R$ es anillo total de fracciones.

\section{Anillo de Hermite}

Si $M$ es un $R$-módulo libre, toda base $\mathcal{B}=$ $\left\{\mathbf{u}_{i}\right\}_{i \in I}$ de $M$ induce un isomorfismo $M \simeq R^{I}$ que asocia a cada $\boldsymbol{a} \in M$ sus coordenadas $\left(a_{i}\right)_{i \in I}$ en la base $\mathcal{B}$.

Definición 3.1. Sean $M$ un $R$-módulo libre y $\mathcal{B}=$ $\left\{\mathbf{u}_{i}\right\}_{i \in I}$ una base de $M$. Definimos $I(\boldsymbol{a})$ como el ideal de $R$ generado por las "coordenadas" de $\boldsymbol{a}$ en $\mathcal{B}$. Es decir, si $\boldsymbol{a}=\sum_{i \in I} a_{i} \mathbf{u}_{i}$, entonces $I(\boldsymbol{a})=$ $\left(\left\{a_{i}\right\}_{i \in I}\right) R=\left(\left\{a_{i}\right\}_{i \in I}\right)$.

Proposición 3.2. $I(\boldsymbol{a})$ es independiente de la base $\mathcal{B}$ elegida.

Demostración. Sea $\mathcal{B}^{\prime}=\left\{\mathbf{v}_{i}\right\}_{i \in I}$ otra base de $M$. Entonces $\mathbf{v}_{i}=\sum_{j \in I} c_{i j} \mathbf{u}_{j}$ donde para todo $i$, \# $(j$ : $\left.c_{i j} \neq 0\right)<\infty$. Luego

$$
\begin{aligned}
\boldsymbol{a} & =\sum_{i \in I} b_{i} \mathbf{v}_{i}=\sum_{i \in I} b_{i} \sum_{j \in I} c_{i j} \mathbf{u}_{j} \\
& =\sum_{j \in I}\left(\sum_{i \in I} c_{i j} b_{i}\right) \mathbf{u}_{j} .
\end{aligned}
$$

Además, como $\boldsymbol{a}=\sum_{j \in I} a_{j} \mathbf{u}_{j}$, entonces $a_{j}=$ $\sum_{i \in I} c_{i j} b_{i}$ y $\left(\left\{a_{j}\right\}_{j \in I}\right) \subset\left(\left\{b_{i}\right\}_{i \in I}\right)$. Por simetría en la prueba tenemos que $\left(\left\{b_{i}\right\}_{i \in I}\right) \subset\left(\left\{a_{j}\right\}_{j \in I}\right)$ y por tanto $\left(\left\{a_{j}\right\}_{j \in I}\right)=\left(\left\{b_{i}\right\}_{i \in I}\right)$.

Definición 3.3. Sea $M$ un $R$-módulo libre de rango finito.

1. Un elemento $\boldsymbol{a} \in M$ se dice unimodular si $I(\boldsymbol{a})=R$.

2. Un elemento $\boldsymbol{a} \in M$ se llama complementable si existe una base de $M$ que contiene a $\boldsymbol{a}$.

3. Un anillo $R$ se llama anillo de Hermite si para todo $M, R$-módulo libre de rango finito, cada elemento unimodular es complementable.

Observación 3.4. Sea $M=R^{n}$.

1. Un vector fila $\left(a_{1}, \ldots, a_{n}\right) \in R^{n}$ es unimodular si y sólo si existen $\lambda_{1}, \ldots, \lambda_{n} \in R$ tales que $\lambda_{1} a_{1}+\cdots+\lambda_{n} a_{n}=1$.

2. Un elemento $\left(a_{1}, \ldots, a_{n}\right) \in R^{n}$ es complementable si y sólo si existe una matriz $A$ invertible de tamaño $n \times n$ cuya primera fila es $\left(a_{1}, \ldots, a_{n}\right)$.
3. Un anillo $R$ es Hermite si y sólo si, para todo $n$, todo vector $\left(a_{1}, \ldots, a_{n}\right) \in R^{n}$ unimodular es complementable.

En 1.976 la conjetura de los anillos de Hermite se enunciaba como (ver [13, 12, 17]): Si $R$ es un anillo de Hermite, entonces $R[x]$ es también un anillo de Hermite. Esta conjetura es equivalente a que si $R$ es un anillo conmutativo y $v=\left(v_{0}(x), \ldots, v_{n}(x)\right)$ es una fila unimodular sobre $R[x]$ tal que $v(0)=(1,0, \ldots, 0)$ entonces $v$ puede ser complementada a una matriz en $G L_{n+1}(R[x])$. Mnif y Yengui en [15] presentan un algoritmo para complementar filas unimodulares sobre anillos noetherianos pero finalmente Yengui en [17] prueba la conjetura en el caso en que $R$ tiene dimensión de Krull menor o igual a 1, y su demostración depende en gran medida de Roitman [16].

Ejemplo 3.5. Un cuerpo y el dominio $\mathbb{Z}$ son ejemplos de anillos de Hermite. Además, el anillo de polinomios $K\left[x_{1}, \ldots, x_{n}\right]$, con $K$ cuerpo, es ejemplo de anillo de Hermite debido al Teorema de Quillen-Suslin.

Veamos un ejemplo de un anillo que no es Hermite [16].

Ejemplo 3.6 (Una fila unimodular que no es complementable). Consideremos el anillo de polinomios reales sobre la 2-esfera $S^{2}$,

$$
R=\frac{\mathbb{R}[x, y, z]}{\left(x^{2}+y^{2}+z^{2}-1\right)} .
$$

Entonces $(\bar{x}, \bar{y}, \bar{z}) \in R^{3}$ es una fila unimodular pues $\bar{x} \bar{x}+\bar{y} \bar{y}+\bar{z} \bar{z}-1=\overline{0}$. Ahora veamos que $(\bar{x}, \bar{y}, \bar{z})$ no es complementable:

Supongamos que $(\bar{x}, \bar{y}, \bar{z})$ es complementable es decir que existe $Q \in G L_{3}(R)$ tal que

$$
Q=\left(\begin{array}{ccc}
\bar{x} & \bar{y} & \bar{z} \\
a_{21} & a_{22} & a_{23} \\
a_{31} & a_{32} & a_{33}
\end{array}\right)
$$

Considere $B=\left(Q^{t}\right)^{-1}$ entonces $B Q^{t}=I_{3} \mathrm{y}$

$$
\begin{aligned}
& \left(\begin{array}{lll}
b_{11} & b_{12} & b_{13} \\
b_{21} & b_{22} & b_{23} \\
b_{31} & b_{32} & b_{33}
\end{array}\right)\left(\begin{array}{lll}
\bar{x} & a_{21} & a_{31} \\
\bar{y} & a_{22} & a_{32} \\
\bar{z} & a_{23} & a_{33}
\end{array}\right) \\
= & \left(\begin{array}{lll}
1 & 0 & 0 \\
0 & 1 & 0 \\
0 & 0 & 1
\end{array}\right)
\end{aligned}
$$


Entonces, la aplicación

$$
\begin{aligned}
\phi: S^{2} & \rightarrow \mathbb{R}^{3} \\
p & \mapsto\left(b_{21}(p), b_{22}(p), b_{23}(p)\right)
\end{aligned}
$$

es un campo vectorial analítico sobre $S^{2}$ tal que nunca se anulan las tres componentes al mismo tiempo, pues es una fila de una matriz invertible y esto no es posible por la topología de $S^{2}$.

En la Proposición 3.7 vemos otro ejemplo importante de anillo de Hermite.

Proposición 3.7. Sea $R$ un anillo local, es decir, $R$ tiene un único ideal maximal. Entonces $R$ es anillo de Hermite.

Demostración. Sea $\mathfrak{m}$ el ideal maximal de $R$. Para toda fila unimodular $\left(a_{1}, \ldots, a_{n}\right) \in R^{n}$ existen $x_{1}, \ldots, x_{n} \in R$ tales que $a_{1} x_{1}+\cdots+a_{n} x_{n}=1$. Entonces el ideal generado por $x_{1}, \ldots, x_{n}$ cumple que $\left(x_{1}, \ldots, x_{n}\right) \nsubseteq \mathfrak{m}$. Esto es, existe $i$ tal que $x_{i} \notin \mathfrak{m}$. Luego $x_{i}$ es invertible, es decir, existe $x_{i}^{-1} \in R$ tal que $x_{i} x_{i}^{-1}=1$. Así, existe una matriz con determinante 1 ,

$$
\left|\begin{array}{cccccccc}
x_{1} & x_{2} & \cdots & x_{i-1} & x_{i} & x_{i+1} & \cdots & x_{n} \\
\lambda & 0 & \cdots & 0 & 0 & 0 & \cdots & 0 \\
0 & 1 & \cdots & 0 & 0 & 0 & \cdots & 0 \\
\vdots & \vdots & & \vdots & \vdots & \vdots & & \vdots \\
0 & 0 & \cdots & 1 & 0 & 0 & \cdots & 0 \\
0 & 0 & \cdots & 0 & 0 & 1 & \cdots & 0 \\
\vdots & \vdots & & \vdots & \vdots & \vdots & & \vdots \\
0 & 0 & \cdots & 0 & 0 & 0 & \cdots & 1
\end{array}\right|=\lambda x_{i} .
$$

Note que $\lambda x_{i}=1$ si $\lambda=(-1)^{i+1} x_{i}^{-1}$. En consecuencia $R$ es un anillo de Hermite.

Observe que un anillo local es Hermite pero no es necesariamente anillo total de fracciones. Por ejemplo, $\mathbb{Z} /(6)$ es un anillo total de fracciones y no es anillo local, de igual forma existen anillos locales que no son anillos totales de fracciones.

Proposición 3.8. Sea $\left\{R_{i}\right\}_{i \in I}$ una familia de anillos de Hermite, entonces $R=\prod_{i \in I} R_{i}$ es anillo de Hermite.

Demostración. Consideremos la proyección $i$-ésima, $\pi_{i}: R \rightarrow R_{i}$. Para toda fila unimodular $\left(\boldsymbol{a}_{1}, \ldots, \boldsymbol{a}_{n}\right) \in R^{n}$, existen $\boldsymbol{b}_{1}, \ldots, \boldsymbol{b}_{n} \in R$ tales que $\boldsymbol{b}_{1} \boldsymbol{a}_{1}+\cdots+\boldsymbol{b}_{n} \boldsymbol{a}_{n}=\mathbf{1}$. Aplicando la proyección $i$-ésima, $\pi_{i}\left(\boldsymbol{b}_{1}\right) \pi_{i}\left(\boldsymbol{a}_{1}\right)+\cdots+\pi_{i}\left(\boldsymbol{b}_{n}\right) \pi_{i}\left(\boldsymbol{a}_{n}\right)=1$, entonces $\left(\pi_{i}\left(\boldsymbol{a}_{1}\right), \ldots, \pi_{i}\left(\boldsymbol{a}_{n}\right)\right)$ es una fila unimodular para todo $i \in I$. Por otra parte, como $R_{i}$ es un anillo de Hermite, existe una matriz invertible $M_{i}=\left(\alpha_{r s}^{i}\right)_{1 \leq r, s \leq n}$ con primera fila $\left(\pi_{i}\left(\boldsymbol{a}_{1}\right), \ldots, \pi_{i}\left(\boldsymbol{a}_{n}\right)\right)$ para todo $i \in I$, por tanto, existe $M=\left(\boldsymbol{\alpha}_{r s}\right)_{1 \leq r, s \leq n}$ invertible con primera fila $\left(\boldsymbol{a}_{1}, \ldots, \boldsymbol{a}_{n}\right)$. En efecto, definiendo $\boldsymbol{\alpha}_{r s}$ como $\pi_{i}\left(\boldsymbol{\alpha}_{r s}\right)=\alpha_{r s}^{i}$ para todo $i \in I$ y como $\pi_{i}$ es un homomorfismo de anillos entonces, para todo $i \in I$,

$$
\begin{aligned}
& \pi_{i}\left(\operatorname{det}\left(\begin{array}{ccc}
\boldsymbol{a}_{1} & \ldots & \boldsymbol{a}_{n} \\
\boldsymbol{\alpha}_{21} & & \boldsymbol{\alpha}_{2 n} \\
\vdots & & \vdots \\
\boldsymbol{\alpha}_{n 1} & & \boldsymbol{\alpha}_{n n}
\end{array}\right)\right) \\
= & \operatorname{det} \pi_{i}\left(\begin{array}{ccc}
\boldsymbol{a}_{1} & \ldots & \boldsymbol{a}_{n} \\
\boldsymbol{\alpha}_{21} & & \boldsymbol{\alpha}_{2 n} \\
\vdots & & \vdots \\
\boldsymbol{\alpha}_{n 1} & & \boldsymbol{\alpha}_{n n}
\end{array}\right) \\
= & \operatorname{det}\left(\begin{array}{ccc}
\pi_{i}\left(\boldsymbol{a}_{1}\right) & \ldots & \pi_{i}\left(\boldsymbol{a}_{n}\right) \\
\alpha_{21}^{i} & & \alpha_{2 n}^{i} \\
\vdots & & \vdots \\
\alpha_{n 1}^{i} & & \alpha_{n n}^{i}
\end{array}\right)=1 .
\end{aligned}
$$

Luego la matriz $M$ tiene determinante uno ya que $\operatorname{det}\left(\boldsymbol{\alpha}_{r s}\right)_{1 \leq r, s \leq n}=\mathbf{1}$ si y sólo si $\pi_{i}\left(\operatorname{det}\left(\boldsymbol{\alpha}_{r s}\right)_{1 \leq r, s \leq n}\right)=1$ para todo $i \in I$. En consecuencia, $R$ es anillo de Hermite.

Corolario 3.9. Un producto directo de cuerpos es anillo de Hermite.

Observación 3.10. Un subanillo de un anillo de Hermite no es en general Hermite y un cociente de un anillo de Hermite tampoco es Hermite. Por ejemplo, el anillo del Ejemplo 3.6 es un dominio entero por tanto su cuerpo de fracciones $\operatorname{Fr}(R)$ es anillo de Hermite, $R \subset \operatorname{Fr}(R)$ y $R$ no es Hermite. Además $\mathbb{R}[x, y, z]$ es un anillo de Hermite y $R=$ $\mathbb{R}[x, y, z] /\left(x^{2}+y^{2}+z^{2}-1\right)$ no lo es.

\section{4. Álgebra finita sobre un cuerpo $K$}

Considere $A$ una $K$-álgebra finita, es decir, $A$ es una álgebra conmutativa con unidad y de dimensión finita como espacio vectorial sobre un cuerpo $K$, su dimensión se denota por $\operatorname{dim}_{K} A$. En [7] hemos estudiado con más detalle las $K$-álgebras finitas. Existen, 
salvo isomorfismos, tres álgebras de dimensión 2 sobre $\mathbb{R}$ :

$$
\mathbb{C}=\frac{\mathbb{R}[x]}{\left(x^{2}+1\right)}, \quad \mathbb{P}=\frac{\mathbb{R}[x]}{\left(x^{2}-1\right)}, \quad \mathbb{D}=\frac{\mathbb{R}[x]}{\left(x^{2}\right)} .
$$

Esto es debido a que en una extensión de grado 2 de $\mathbb{R}$,

$$
A=\frac{\mathbb{R}[x]}{\left(x^{2}+b x+c\right)}
$$

se pueden dar tres casos según $x^{2}+b x+c$ tenga dos raíces imaginarias, dos raíces reales distintas o una raíz doble. Los conjuntos $\mathbb{C}, \mathbb{P}, \mathbb{D}$ son, respectivamente, los números complejos, los números paracomplejos y los números duales. Note que $\mathbb{C}$ es un cuerpo, mientras que $\mathbb{P}$ y $\mathbb{D}$ no lo son pues $\mathbb{P}$ tiene divisores de cero y $\mathbb{D}$ tiene además elementos nilpotentes.

Las rectas proyectivas sobre las $\mathbb{R}$-álgebras $\mathbb{C}, \mathbb{P}, \mathbb{D}$ generan las tres geometrías clásicas del plano, Moebius, Laguerre y Minkowski, ver [9]. Un problema abierto en geometría proyectiva es caracterizar la recta proyectiva sobre anillos. Existen trabajos recientes sobre este tema pero en general es una teoría muy incompleta. En [11] se presenta un trabajo sobre la geometría correspondiente a la $\mathbb{R}$-álgebra tridimensional $\frac{\mathbb{R}[x]}{\left(x^{3}\right)}$ y [6] es un estudio inicial de las rectas proyectivas sobre anillos totales de fracciones.

Proposición 4.1. ([7], Proposición 2.2). $A$ es una $K$-álgebra finita si y sólo si $A$ es una suma directa de $K$-álgebras finitas locales.

Proposición 4.2. Toda $K$-álgebra finita es un anillo total de fracciones.

Demostración. Sean $A$ una $K$-álgebra finita y $\operatorname{dim}_{K} A=n$. Para todo $u \in A$, existe $r<n$ tal que $1, u, \ldots, u^{r}$ son linealmente independientes y $u^{r+1}$ depende linealmente de $\left\{1, u, \ldots, u^{r}\right\}$ luego existen $b_{0}, b_{1}, \ldots, b_{r} \in K$ tales que $u^{r+1}=b_{r} u^{r}+\cdots+b_{0} 1$ y tenemos dos casos:

1. Si $b_{0}=0$ entonces $0=u^{r+1}-b_{r} u^{r}-\cdots-$ $b_{1} u=u\left(u^{r}-\cdots-b_{2} u-b_{1} 1\right)$, luego, $u$ es divisor de cero. Note que $u^{r}-\cdots-b_{2} u-b_{1} 1 \neq 0$ ya que $1, u, \ldots, u^{r}$ son linealmente independientes.

2. Si $b_{0} \neq 0$ entonces $1=b_{0}^{-1} u\left(u^{r}-\cdots-b_{2} u-\right.$ $\left.b_{1} 1\right)$, por tanto, $u$ es invertible.

En consecuencia, $A$ es un anillo total de fracciones.
El ejemplo más simple de las $K$-álgebras finitas es el de las $K$-álgebras $A=\frac{K[x]}{(f(x))}$ caracterizadas por la existencia de $u \in A$ tal que $1, u, \ldots, u^{n-1}$ es base de $A$ como $K$-espacio vectorial. Obviamente no toda $K$-álgebra finita es de este tipo, por ejemplo, en $A=\frac{\mathbb{R}[x, y]}{(x, y)^{2}}$ todo elemento al cuadrado es cero, luego no puede existir $u \in A$ tal que $1, u, u^{2}$ sea base de $A$ como $K$-espacio vectorial. El siguiente es ejemplo de anillo total de fracciones y anillo de Hermite que no es $\mathbb{R}$-álgebra finita.

Ejemplo 4.3. (Un anillo total de fracciones con maximal no nilpotente)

Sea $\mathbb{R}[[x, y]]$ el anillo de series de potencias con coeficientes en $\mathbb{R}$. Consideremos el anillo

$$
\begin{aligned}
R & =\frac{\mathbb{R}[[x, y]]}{(x(x+y), y(x+y))} \\
& =\{a+b \bar{y}+\bar{x} s(\bar{x}): a, b \in \mathbb{R} \text { y } s(\bar{x}) \in \mathbb{R}[[\bar{x}]]\}
\end{aligned}
$$

Note que los elementos de $R$ satisfacen que $\bar{x}^{2}=$ $\bar{y}^{2}=-\overline{x y}$ y en general para cada $r \geq 2, \bar{x}^{r}=$ $(-1)^{s} \bar{y}^{s} \bar{x}^{r-s}$ para todo $s=1, \ldots, r$. El producto de dos elementos en $R$ es

$$
\begin{aligned}
& (a+b \bar{y}+\bar{x} s(\bar{x}))(c+d \bar{y}+\bar{x} t(\bar{x})) \\
= & a c+(a d+b c) \bar{y}+\bar{x} h(\bar{x}),
\end{aligned}
$$

donde $h(\bar{x}) \in \mathbb{R}[[\bar{x}]]$. Veamos que $R$ es un anillo total de fracciones.

1. Si $a \neq 0$ entonces $a+b \bar{y}+\bar{x} s(\bar{x})$ es invertible. En efecto, por la fórmula del producto,

$$
\begin{aligned}
& (a+b \bar{y}+\bar{x} s(\bar{x}))(a-b \bar{y}+\bar{x} s(\bar{x})) \\
= & (a+\bar{x} s(\bar{x}))^{2}-b^{2} \bar{y}^{2} \\
= & (a+\bar{x} s(\bar{x}))^{2}-b^{2} \bar{x}^{2} \\
= & a^{2}+\bar{x} h(\bar{x})
\end{aligned}
$$

donde $h(\bar{x})=2 a s(\bar{x})+\bar{x}(s(\bar{x}))^{2}-b^{2} \bar{x}$. Note que $a^{2}+\bar{x} h(\bar{x}) \in \mathbb{R}[[\bar{x}]]$ es invertible ya que $a \neq 0$ luego existe $r(\bar{x}) \in \mathbb{R}[[\bar{x}]]$ tal que $(a+b \bar{y}+\bar{x} s(\bar{x}))(a-b \bar{y}+\bar{x} s(\bar{x})) r(\bar{x})=1$. En consecuencia, $a+b \bar{y}+\bar{x} s(\bar{x})$ es invertible.

2. Si $a=0$ entonces $a+b \bar{y}+\bar{x} s(\bar{x})$ es un divisor de cero. En efecto, si $a=0$ entonces existe $\bar{x}+\bar{y} \in R$ tal que $(b \bar{y}+\bar{x} s(\bar{x}))(\bar{x}+\bar{y})=0$.

Note que $R$ es anillo local con ideal maximal $(\bar{x}, \bar{y})$ pues está formado por las no unidades de $R$. Además, $R$ no es nilpotente pues si lo fuera existiría $n \in \mathbb{N}$ 138 
tal que $(\bar{x}, \bar{y})^{n}$ y en particular existiría $n \in \mathbb{N}$ tal que $\bar{x}^{n}=0$. Como $\bar{x}=x+(x(x+y), y(x+y))$ entonces $\bar{x}^{n}=x^{n}+(x(x+y), y(x+y))=0$ esto es $x^{n} \in(x(x+y), y(x+y))$. Luego $x+y$ divide a $x^{n}$ y esto es absurdo. En consecuencia, $R$ es un anillo total de fracciones y anillo local pero no es $\mathbb{R}$-álgebra finita. Además, por la Proposición 3.7, $R$ es anillo de Hermite.

Proposición 4.4. Toda $K$-álgebra finita es un anillo de Hermite.

Demostración. Sea $A$ una $K$-álgebra finita. Por la Proposición 4.1, existen $A_{1}, \ldots, A_{r} K$-álgebras locales finitas tales que $A=A_{1} \times \cdots \times A_{r}$. Puesto que $A_{1}, \ldots, A_{r}$ son anillos locales, por la Proposición 3.7, $A_{1}, \ldots, A_{r}$ son anillos de Hermite y por la Proposición 3.8, el producto de anillos de Hermite es un anillo de Hermite. En consecuencia, $A$ es un anillo de Hermite.

\section{Conclusiones}

En este artículo se han estudiado propiedades generales de anillos totales de fracciones y anillos de Hermite. Se consideran las $K$-álgebras finitas para probar que estas son anillos totales de fracciones y anillos de Hermite. Además, se mostró que el producto directo de cuerpos es también un anillo total de fracciones y anillo de Hermite.

La relación del anillo total de fracciones con otros anillos es un tema de investigación en la actualidad en álgebra conmutativa [2, 10]. Además, resolver la conjetura de los anillos de Hermite es de interés permanente entre los investigadores [12, 13, 15, 16, 17]. Nuestro interés en estos dos anillos se basa en un problema abierto en geometría proyectiva, el cual consiste en caracterizar la recta proyectiva sobre anillos [6, 9, 11]. Existen trabajos recientes sobre este tema pero en general es una teoría muy incompleta. [11] es un trabajo sobre la geometría correspondiente a la $\mathbb{R}$-álgebra tridimensional $\frac{\mathbb{R}[x]}{\left(x^{3}\right)}$. Más exactamente, queremos caracterizar las rectas proyectivas sobre anillos totales de fracciones y anillos de Hermite. Para alcanzar este fin, se han estudiado las $K$-álgebras finitas en [7] y [6] es un estudio prelimimar de la recta proyectiva sobre anillos totales de fracciones.

\section{Agradecimientos}

Los autores desean agradecer a los árbitros de la revista por sus comentarios y sugerencias.

\section{Referencias}

[1] F. Anderson and K. Fuller, Rings and Categories of Modules, Second Edition, Springer-Verlag, New York, 1992. 2. 5

[2] M. Arapovic, "Characterizations of the 0dimensional rings", Glasnik Matematicki, vol. 18, no. 38, pp. 39-46, 1983. 2, 9

[3] M.F. Atiyah y I.G. Macdonald, Introducción al álgebra conmutativa, Editorial Reverté S. A., Barcelona, 1980. 2

[4] N. Bourbaki, Commutative algebra, SpringerVerlag, Translated from the french, Reprint of the 1972 edition, 1989. 2

[5] D. Eisenbud, Commutative Algebra, with a view toward Algebraic Geometry, Springer-Verlag, New York, 1995. 2

[6] C. Granados Pinzón, Tesis doctoral: Álgebras finitas sobre un cuerpo. La recta proyectiva, Universidad de Valladolid, Valladolid, 2015. 2, 8, 9

[7] C. Granados Pinzón y W. Olaya León, " $K$-álgebras finitas conmutativas con unidad, Ingeniería y Ciencia", vol. 12, no. 24, pp. 31-49, 2016. 2, 5, 7, 8, 9

[8] C. Granados Pinzón, W. Olaya León y S. Pinzón, "Estimación del cardinal del espectro maximal de un producto de cuerpos", Ciencia en Desarrollo, vol. 9, no. 2, pp. 83-93, 2018. 5

[9] E. Hartmann, "Planar Circle Geometries: an introduction to Moebius-, Laguerreand Minkowski-planes", Darmstadt University of Technology, 2004. Disponible en: http://www.mathematik.tudarmstadt.de/ ehartmann/circlegeom-introd.pdf 2.,8, 9

[10] M. Hashimoto, "Equivariant total ring of fractions and factoriality of rings generated by semiinvariants", Communications in Algebra 43, pp. 1524-1562, 2015. 2, 9

[11] H. Havlicek and K. List, "A three-Dimensional Laguerre geometry and its visualization, In proceedinhs-Dresden Symposium geometry: 
constructive and kinematic", Institut für geometrie TU Dresden, Dresden pp. 122-129, 2003. arXiv:1304.0223v1 [math.AG] 31 Mar 2013. 2. 8,9

[12] T.Y. Lam, Serre's Problem on Projective Modules, Springer Monographs in Mathematics, 2006. 2, 6, 9

[13] T.Y. Lam, Serre's conjecture, Lecture notes in mathematics 635, Springer-Verlag, Berlin-New York, 1978. 2, 6, 9

[14] H. Matsumura, Commutative Ring Theory, Cam- bridge University Press, Cambridge, 1989. 2

[15] A. Mnif and I. Yengui, "An algorithm for unimodular completion over Noetherian rings", J. Algebra 316, pp. 483-498, 2007. 2, 6, 9

[16] M. Roitman, "Completing Unimodular Rows to Invertible Matrices", J. Algebra 49, pp. 206-211, 1977. 2, 6, 9

[17] I. Yengui, "The Hermite ring conjecture in dimension one", J. Algebra 320, pp. 437-441, 2008. 2, 6, 9 